\title{
Revisión sistémica de artículos de capital intelectual como generador de innovación en turismo de salud
}

\author{
Sonia Guadalupe Zermeño-Flores \\ Universidad Estatal de Sonora \\ Tomás Cuevas-Contreras \\ Universidad Autónoma de Ciudad Juárez
}

\section{Resumen}

Con el fin de elaborar una revisión sistémica (Rs) de algunas fuentes de información, el presente documento analiza y evalúa 40 artículos sobre dos variables de investigación que se propone relacionar: el capital intelectual y la innovación en el turismo de salud y 45 referencias de definiciones de estas variables. Se busca responder cuestionamientos como los siguientes: ¿hacia cuáles temáticas se orientan los trabajos?; ¿qué tipo de textos son (ensayos, investigaciones aplicadas, análisis de literatura, entre otras)?; ¿cómo enfocan las definiciones de las variables empleadas?; ¿en qué teorías se sustentan?; ¿qué método de estudio utilizan?; iqué instrumentos de recolección de datos se eligieron para las investigaciones aplicadas? De los 40 documentos, 25 son sobre la variable capital intelectual y 15 sobre innovación, dado que su contenido responde a los criterios de elegibilidad en cuanto factor de impacto, actualidad, enfoque, idioma, entre otros indicadores de fiabilidad y validez.

\section{Palabras clave}

Revisión sistémica, capital intelectual, innovación, clúster, turismo de salud.

Recibido: 03/12/2014· Aceptado: 04/06/2015

*Correos electrónicos:soniagpez@gmail.com, tcuevas@uacj.mx. 


\title{
Systemic review of articles about intellectual capital as source innovation in health tourism
}

\author{
Sonia Guadalupe Zermeño-Flores \\ Universidad Estatal de Sonora \\ Tomás Cuevas-Contreras \\ Universidad Autónoma de Ciudad Juárez
}

\begin{abstract}
This paper is an analysis and evaluation of articles that allows Systemic review (sr) sources of information related with two variables proposed research associate: intellectual capital and innovation in the health tourism. In reviewing these articles seeks to answer questions like: what are the issues in which the works are oriented?; What kind of writing are (essays, applied research, literature review, etc.)?; How focused the definitions of the variables worked?; What theories are based?; What method of study used?; What data collection instruments, chosen for applied research? 40 documents were reviewed, 25 articles of Intellectual Capital and 15 at innovation, because its content is desirable for the interests of the review and meet the eligibility criteria as impact factor, actuality, focus, language, among other indicators of reliability and validity, but are limited.
\end{abstract}

\section{KEY WORDS}

Systemic review, intellectual capital, innovation, cluster, health tourism. 


\section{Introducción}

El conocimiento es uno de los principales elementos que fomenta la innovación y es parte esencial del capital intelectual (cr) humano. La gestión de este capital parte de las intelectualidades, la experiencia, el talento, las habilidades comunicativas y las destrezas que posee y que se gestan en el recurso humano de las organizaciones. En este sentido, la organización se convierte a su vez en productora de conocimientos y fomenta comportamientos o cultura de colaboración, iniciativa y compromiso, que serán ingredientes básicos para la creación de estrategias innovadoras para conformar ventajas competitivas sostenibles.

Varios autores han trabajado con el binomio conocimiento-innovación (Perozo, 2004; Nagles, 2007; Darceles, 2007; Estrada y Dutrénit, 2007; Muñoz, Aguado y Lucía, 2003; Rivas y Flores, 2007; Heredia, 2009; Magaña, 2014; Hoarau, Hindertje y Kline, 2014), otros se han dedicado a monitorear solo una variable: capital intelectual, para señalar métodos de valuación del capital dentro de las organizaciones (Inche y Álvarez, 2007; Funes y Hernández, 2001; García, 2005; Madrigal, 2009; Bontis, 1998 a; Alizadeh, 2012); algunos estudios más se enfocan en la creación de clústeres como estrategias de innovación (Ivars, Vera y Acebal, 2014; Carlisle et al., 2013; Novelli, Schmitz y Spencer, 2006).

Para analizar la literatura citada pueden utilizarse las revisiones sistémicas (RS), entendidas como la revisión de una pregunta formulada con claridad por medio de métodos sistemáticos y explícitos para identificar, seleccionar y valorar críticamente investigaciones relevantes sobre tal pregunta, así como para recoger y analizar datos de los estudios incluidos en la revisión (Marín, Tobías y Seoane, 2006, citado en Sánchez-Meca y Botella, 2010). La Rs surge como un intento de salvar las limitaciones de las revisiones tradicionales, caracterizadas por ser cualitativas y carecer de una adecuada sistematización.

El trabajo de revisión de literatura va más allá de leer un artículo o extraer definiciones o ciertos datos, es un acto de análisis y de valoración de la información, de la validez de las fuentes, del apego a una estructura estandarizada de redacción que permite localizar datos de manera más rápida y eficiente.

Así, se presenta una revisión sistémica de tipo cualitativo que pretende identificar los rasgos comunes y las diferencias entre los distintos artículos revisados en relación con su enfoque, sus objetivos, teorías, método, instrumento de recolección de datos y resultados obtenidos. 


\section{Método}

Las Rs, y en especial los metaanálisis, son investigaciones científicas cuya finalidad es integrar de forma objetiva y sistémica los resultados de los estudios empíricos sobre un determinado problema de investigación, con el fin de establecer el estado del arte en un campo de estudio concreto. Se desarrollan en etapas como: 1) formulación del problema, 2) definición de criterios de inclusión y búsqueda de los estudios, 3) codificación de las características de los estudios que puedan moderar los resultados, 4) cálculo del tamaño del efecto, 5) técnicas de análisis estadístico e interpretación, y 6) publicación (Sánchez-

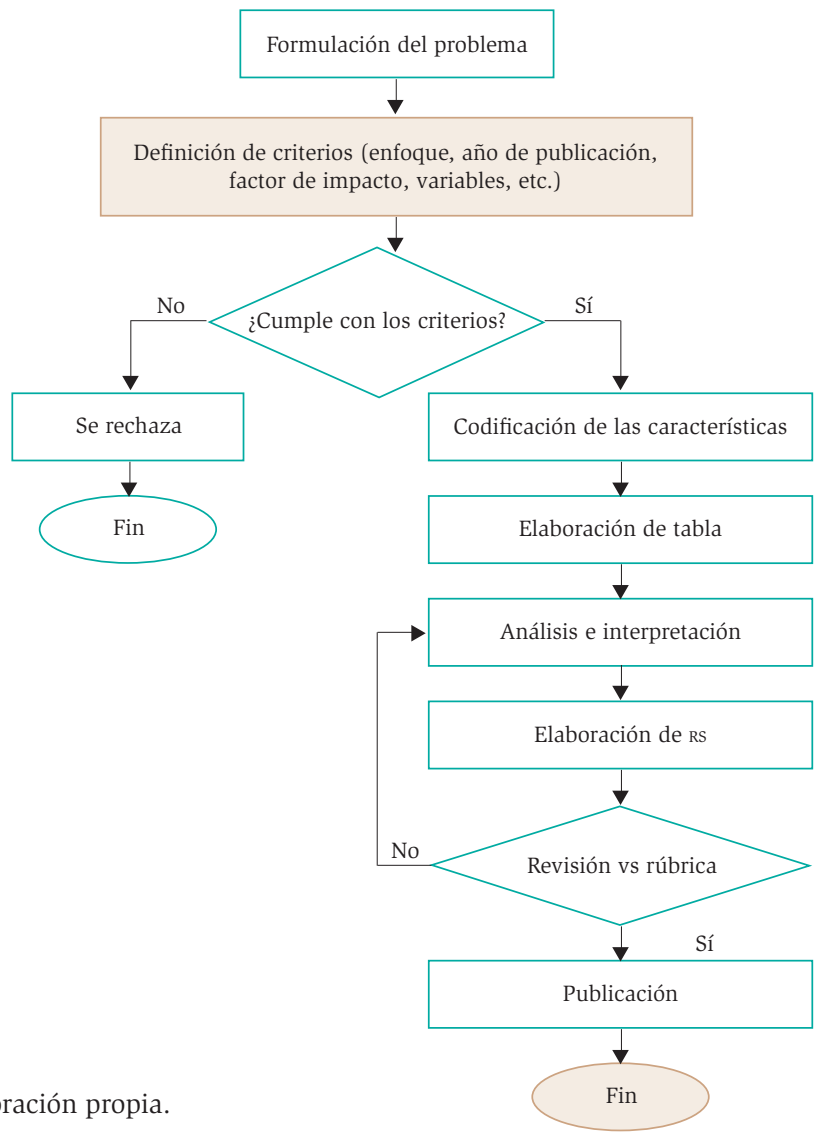

Figura 1. Procedimiento para la elaboración de revisiones sistémicas 
Meca y Botella, 2010). Con estos criterios del procedimiento se elabora la siguiente revisión sistémica de literatura, la cual busca ser una guía que apoye a las revisiones sistémicas posteriores, aunque no tenga un protocolo de registro actualizado que ampare el trabajo de análisis (figura 1).

Por medio del procedimiento anterior, de las listas de verificación de componentes que nos proporcionan la metodología Strengthening the Reporting of Observational Studies in Epidemiology (sтRовE) (Vandenbrouck et al., 2009) y la declaración Preferred Reporting Items for Systematic reviews and MetaAnalyses (PRISMA) (Urrútia y Bonfill, 2010) para el análisis de revisiones sistémicas y los metaanálisis, el presente escrito tiene como objetivo analizar investigaciones aplicadas sobre las variables capital intelectual e innovación para determinar los rasgos comunes y las diferencias entre los distintos artículos, en relación con sus enfoques, análisis de las definiciones, características, teorías y modelos que los sustentan, objetivos que persiguen, métodos utilizados para la investigación, instrumentos de recolección de datos y resultados obtenidos.

Para localizar y elegir los trabajos se recomienda ser organizado desde un principio a fin de mantener bien establecidas las variables por investigar, los propósitos y las bases más adecuadas que generen información actualizada, procedente, fiable y de alto impacto. Las bases de datos utilizadas para este trabajo incluyeron aquellas que estaban en lengua castellana, como es el caso de Redalyc, además de otras como:

http://www.erevistas.csic.es

http://www.ejbrm.com

http://www.recolecta.net

http://doaj.org/

http://dialnet.unirioja.es/

http://www.theibfr.com http://www.conricyt.mx

http://publicaciones.eafit.edu.co

http://eprints.rclis.org

http://www.revistarecai.mx

http://www.opendoar.org

http://www.revistaeggp.uchile.cl/

http://estatico.uned.ac.cr/

Si bien es cierto que los artículos permitieron entender mejor el tema y sus alcances, muy pocos fueron de investigaciones aplicadas.

El mecanismo de búsqueda fue más organizado en la segunda etapa; además de las variables estudiadas (capital intelectual, innovación y su relación con el turismo de salud), se identificaron los posibles enfoques teóricos (manejo de los clústeres y los stakeholders). La búsqueda se realizó en bases de datos más 
calificadas y con estudios de otros países, además de América Latina y España, como: Web of Science, Elsevier, Emerald, Google Academic, entre otras. Las palabras de búsqueda fueron: intellectual capital, innovation, medical tourism, clusters y stakeholders.

Los artículos se eligieron con base en el año de publicación (de 2000 en adelante, preferentemente), las palabras clave (gestión del conocimiento, capital intelectual, innovación, innovación en turismo, clústeres), el objetivo del artículo (capital intelectual e innovación en el turismo de salud y como ventaja competitiva, principalmente), el tipo de artículo (investigaciones aplicadas, ensayos, notas del editor, análisis de literatura) y, sobre todo, en el caso de las investigaciones aplicadas, la metodología que habían utilizado, los resultados y la amplitud de la bibliografía.

Para la elección se consideró la importancia del material que puede extraerse de estos trabajos, aunque existe un porcentaje de sesgo porque una buena parte de los artículos no se incluye en revistas con factor de impacto, además de que $60 \%$ de los artículos de innovación tiene una clara tendencia a la aplicación en empresas de turismo.

Con estos datos se desarrolló una tabla matriz de fuentes de información que incluyó columnas con el título del artículo, autor(es), nombre de la revista, volumen, número, páginas del artículo, año de publicación, fecha en que se recuperó, base de datos donde se obtuvo, palabra(s) clave(s) utilizada(s) para la búsqueda, factor de impacto de la revista, palabras clave del artículo, el tipo de escrito, perspectivas teóricas o modelos, componentes o características de la variable trabajada, el tipo de investigación y los instrumentos de recolección de datos utilizados, así como las dimensiones elegidas para el instrumento de obtención de la información.

De este modo, se obtuvo información relevante de los artículos de investigación, ensayos, escritos de editor, entre otros, pero una buena parte de ellos no cumplió con los criterios establecidos. Sin embargo, mostraron información destacada que apoya el ejercicio de desglose temático y ejemplos de cómo se pueden redactar los trabajos académicos. La mayoría de los textos están escritos en inglés, seguidos de 40\% publicados en español, portugués y catalán. 


\section{Resultados}

Del total de investigaciones elegidas, 25 fueron estudios relacionados con la variable capital intelectual y 15 con la de innovación. De ellos se desprendieron los objetivos, las teorías y modelos y los tipos de investigación e instrumentos de recolección de datos utilizados.

\section{Análisis de las definiciones}

Tras revisar las referencias de las definiciones de las variables, se elaboró una tabla de análisis de definiciones que incluyó los nombres de los autores y el año de publicación de la definición. Se analizó cada una de ellas y se separaron por características y elementos comunes. Para realizar un escrito que resumiera y examinara la evolución y los enfoques de los enunciados de las variables se ejecutó una lectura y revisión de los elementos comunes, de tal forma que se marcó con un color las definiciones que se formulaban en un mismo sentido; por ejemplo, las de innovación que hacen referencia a la invención de maquinaria, las que se dirigen a la innovación en los procesos, las que aluden a las ideas, a la creación de nuevos tipos de negocios o las que se describen en la introducción de cambios incrementales y radicales.

Con esta tabla fue posible establecer que el término capital intelectual surgió a principios de los años noventa del siglo xx en Estados Unidos y en Suecia, principalmente, y que mide el valor del conocimiento de la empresa en sus distintos ámbitos: las personas (inteligencia humana), la organización (el know-how de la empresa, las patentes y las marcas) y el mercado (satisfacción de una cartera de clientes). El origen del término capital intelectual se distingue desde tres ámbitos principales: el que nace de la gestión del conocimiento como parte de los recursos tácitos (Dierickx y Cool, 1989, citado en Alizadeh, 2012; Stewart, 1997; Hall, 1992; Klein y Prusak, 1994; Nonaka y Takeuchi, 1995; Sveiby, 1997; Klein, 1998; Ulrich, 1998; Edvinsson y Malone, 1998), el de la esfera contable-financiera, como rubro indispensable en los estados financieros (Brooking, 1997; Roos et al., 1997; The Society of Management Accountants of Canada, 1998 (citado en Dzinkowski, R., 1998); Edvinsson y Malone, 1998; Seetharaman, 2002, citado en Alizadeh, 2012; García-Ayuso y Larrinaga, 2004), y una visión más reciente que retoma el origen del conocimiento dirigido a la 
contribución de ventajas competitivas (Youndt, Subramaniam y Snell, 2004; Chen, Cheng y Hwang, 2005; Viedma, 2007).

Se puede concluir que el ci es un activo intangible de las organizaciones que incluye conocimientos, habilidades, experiencias, datos, propiedad intelectual, bases de datos, procedimientos, cultura organizacional, entre otros, $\mathrm{y}$ que puede clasificarse en capital humano, relacional, estructural y social, a través de cuya gestión pueden generarse innovaciones y ventajas competitivas sostenibles que repercutan en rendimientos para el empleado, la empresa y la sociedad en general.

Por su parte, el término innovación se ha utilizado en diferentes ámbitos sociales y económicos como garantía de competitividad en las empresas; Medina y Espinosa (1994) indican que proviene del latín innovatio (acción y efecto de innovar), e innovar de innovare (cambiar o alterar las cosas incorporándole algo nuevo).

A finales del siglo xviII, con el nacimiento de la producción industrial, la innovación se centró en la creación de máquinas (Smith, 1994). A principios del siglo xx, con los trabajos de Schumpeter (1939), se incorpora el acto de innovar no solo referido a la maquinaria, sino a la mayoría de los procesos. En las décadas de los ochenta y noventa, sobre todo, el concepto se amplía para incorporar las ideas aplicadas a los procesos y como medios y oportunidad para la creación de nuevos negocios. Son ejemplos las definiciones de Van de Ven (1986), Damanpour (1990), Chen y Tsou (2007), Drucker (1985), Tushman y Nadler (1986), Damanpour y Gopalakrishnan (1997). En el nuevo siglo, la innovación se emplea en el ámbito interno, relacionada con procesos complejos de desarrollo tecnológico, de la gestión del conocimiento y las capacidades organizativas, para conseguir competitividad y riqueza, tanto para sus creadores como para las organizaciones, así como para elevar la calidad de vida de la sociedad. Son ejemplo las aportaciones de Santamaría (2001, citado en Trillo y Pedraza, 2011), Johannessen, Olsen y Lumpkin (2011), Fundación Cotec (2002), McElroy (2002), Navarro (2005), Fagerberg, Mowery y Nelson (2006), Euroestat-ocdE (2006), Valhondo (2004), Nagles (2007), Blichfeldt (2009).

Se puede concluir que la innovación es el proceso de introducción de un cambio o mejora en los productos y/o servicios, los procesos, prácticas mercadológicas u organizativas con el fin de hacer más eficiente el trabajo y alcanzar el desarrollo económico de la empresa y la sociedad en general. 


\section{Objetivos de los trabajos}

Los trabajos de capital intelectual se proponen como un elemento de la gestión del conocimiento. Cuatro artículos hacen una revisión de los elementos que conforman el cr (Sánchez, Melián y Hormiga, 2007; Bontis, Chua y Richardson, 2000; Di Domenico, De Bona y Fernández, 2003; González, 2002). Otros cinco analizan los componentes necesarios para medir el cr en las organizaciones (Alizadeh, 2012; Bontis, 1998 b; Funes y Hernández, 2001; García, 2005; Madrigal, 2009). Por su parte, trabajos como los de Marulanda y López (2013), Ochoa, Prieto y Santidrián (2012) y Nagles (2007) revisan los modelos de análisis del cr en las empresas. Cinco trabajos más revisan el componente de ci como una capacidad de gestión interna que apoya la cultura organizacional (Amaya, Iriarte y Perozo, 2006; Orozco, Chavarro-Bohórquez y Rivera, 2007; González, 2002; Perozo, 2004; Muñoz, Aguado y Lucía, 2003). Otros tres artículos se refieren al cı como fuente de ventajas competitivas (Estrada y Dutrénit, 2007; Heredia, 2009; Huang et al., 2008).

La gestión del ci tiene una fuerte tendencia a la mejora continua, ya sea hacia los procesos internos de la firma o hacia el fortalecimiento de las relaciones con los entes externos; por esto los elementos básicos para la formación de estrategias de innovación en los procesos son la organización, la generación de productos y servicios, de los procesos mercadológicos, entre otros. Otros trabajos enlazan la variable ci con la innovación (Correa, Rodrigues y Pereira, 2007; Inche y Álvarez, 2007; Martínez, 2004; Nagles, 2006).

De la misma forma que se analizaron los trabajos de ci, se revisaron los estudios sobre innovación, los cuales muestran dos tendencias principales: la innovación creadora de ventajas competitivas sostenibles y el enfoque principal entre innovación y turismo. Se revisan ahora los trabajo sobre innovación, donde se muestran los trabajos de Carlisle et al. (2013) y Darceles (2007). En cuanto al segundo enfoque se revisaron los trabajos de Hoarau y Kline (2014), Ivars, Vera y Acebal (2014); Magaña (2014); Joukes, Lourenço-Gomes y MartaCosta (2013); Yeoh, Othman y Ahmad (2013); Thomas y Wood (2014); Novelli, Schmitz y Spencer (2006); Richards (2011) y las investigaciones de Chong y Zhou (2014). 


\section{Características del capital intelectual y la innovación}

En cuanto a la determinación de los rasgos que caracterizan al ci y a la innovación se tiene lo siguiente: entre los autores que citan el conocimiento y en general el ci como fuente de ventaja competitiva sostenible están Nagles (2007), Marulanda y López (2013), González (2002), Estrada y Dutrénit (2007), Muñoz, Aguado y Lucía (2003); Ochoa, Prieto y Santidrián (2012); Rivas y Flores (2007); García (2005); Heredia (2009); Orozco, Chavarro-Bohórquez y Rivera (2007). Estos últimos además señalan que el ci se trata de un generador de innovación para la competitividad; así, el conocimiento es el componente clave en la ventaja competitiva porque, como activo, guarda unas propiedades que hacen indefectible una organización. Nagles (2007) añade que la utilización del cI permite resolver problemas, tomar decisiones, agregar valor a los productos y servicios, enriquecer los puestos de trabajo y empoderar a los empleados para asegurar la creación de capacidades que permitan explotar los recursos de la empresa de forma efectiva. Por su parte, Darceles (2007) dirige el cr al uso de la creatividad para generar innovaciones, de esta forma quedan enlazadas las variables y los enfoques de su trabajo de investigación.

De acuerdo con Ochoa, Prieto y Santidrián (2012); Orozco, Chavarro-Bohórquez y Rivera (2007); Estrada y Dutrénit (2007), Heredia (2009) y Alizadeh (2012) el capital intelectual se caracteriza por ser un conjunto de recursos intangibles, o recursos intelectuales destinados para crear valor, únicos, difíciles de imitar, de naturaleza tácita y complejos. Varios autores caracterizan el ci como parte fundamental del valor contable de las empresas (Brooking, 1997; Roos et al., 1997; Edvinsson y Malone, 1998; García-Ayuso y Larrinaga, 2004; García, 2005; Alizadeh, 2012), poniendo de relieve que los activos intangibles deben estar presentes en los informes contables de las organizaciones, por lo que el factor clave es identificar y cuantificar el capital intelectual.

En lo referente a la variable innovación, se plantea que su adopción es gradual, aunque a veces irrumpe de forma disruptiva en las organizaciones, mejorando productos, procesos, estrategias, ideas, tecnología, prácticas en los suministros, entre otras cosas. La innovación se caracteriza por su capacidad para generar soluciones ingeniosas, rentables y creativas, de manera que atiendan las necesidades de un mercado. Innovar se convierte entonces en un factor fundamental para la competitividad de las empresas (Perozo, 2004; Ivars, Vera y Acebal, 2014; Richards, 2011). 
La innovación no es el acto conductor, sino el resultado de aplicar un proceso (Hoarau y Kline, 2014), en este caso de gestión del capital intelectual. Como señala Nagles (2007), desarrollar la innovación es un proceso enfocado a la creación de valor. Desde este punto de vista, la innovación comprende cuatro elementos: producción de un nuevo conocimiento, organización del mismo, su adaptación y, finalmente, la innovación. Asimismo, la innovación es gradual, por lo que las mejoras continuas a menudo se definen como innovaciones incrementales y las revoluciones tecnológicas como innovaciones radicales (Hoarau y Kline, 2014; Schumpeter, 1939, citado en Carlisle et al., 2013).

Aunque no existen características distintivas y determinantes (pues las innovaciones son múltiples, de diversa magnitud y de diferente orden), se puede precisar que se trata de una explotación de recursos, que se maneja en procesos, que puede ser de finalidad tecnológica en el sentido genérico de la palabra, que se efectúa de manera incremental y que busca mejorar las formas de llevar a cabo una actividad o crear nuevos productos y servicios para las demandas de una sociedad.

\section{Componentes de las variables}

Los elementos que conforman el capital intelectual van desde el intelecto hasta los procesos y las relaciones con los entes externos. Sin embargo, Perozo (2004), González (2002), Dárceles (2007), Estrada y Dutrénit (2007) y Madrigal (2009) tienden a privilegiar el componente humano como indispensable del cr.

Marulanda y López (2013) y Marr (2004), indican otros componentes del capital intelectual: los recursos humanos, las relaciones, la infraestructura física, las prácticas y rutinas y la propiedad intelectual.

Edvinsson y Malone (2006, citado en Marulanda y López, 2013), Estrada y Dutrénit (2007), Muñoz, Aguado y Lucía (2003); Inche y Álvarez (2007); Funes y Hernández (2001); Bontis, Chua y Richardson (2000); García (2005); Heredia (2009); Madrigal (2009); González-Loureiro y Figueroa (2013) indican que el CI se forma por el capital humano, el capital estructural y el capital relacional.

Para Amaya, Iriarte y Perozo (2006) los componentes del cr son una red de recursos humanos, de la información, así como informáticos y telemáticos. A su vez, Funes y Hernández (2001) incluyen varias perspectivas para indicar 
sus componentes o tipos. Por ejemplo, citan a Brooking (1997), quien divide el cr de una empresa en cuatro categorías de intangibles: a) activos de mercado, b) activos de propiedad intelectual, c) activos centrados en el individuo, $y$ d) activos de infraestructura.

Con un enfoque propiamente contable del cI, Alizadeh (2012) divide los activos en dos categorías: los activos físicos y financieros y los activos intangibles (la propiedad intelectual, derechos de autor, patentes, el goodwill), es decir, lo que propiamente se indica como capital intelectual.

Autores como Hoarau y Kline (2014) señalan cinco categorías de innovaciones: 1) en los productos, 2) en los procesos, 3) de gestión, 4) en mercadotecnia, y 5) innovaciones. Schumpeter (1939) incorpora la innovación sobre compras y suministros y la introducción en nuevos mercados. La Organización para la Cooperación y el Desarrollo Económicos, en su Manual de Oslo (2006, citado en Mercado, Demuner y Fierro, 2012), incorpora, además de la innovación de productos, de procesos y de organización, la innovación en mercadotecnia.

Para Hjalager (2002, citado en Novelli, Schmitz y Spencer, 2006), subdivide la innovación en: innovaciones de producción y en los procesos, pero incorpora una visión nueva, con las innovaciones de manejo de la información, innovaciones administrativas y las institucionales.

Como puede observarse, los componentes de la innovación son variados, pero la mayoría coincide en argumentar la preponderancia de innovaciones en productos (ya sean bienes o servicios), en procesos, en sistemas organizacionales, en estrategias de comercialización y, en general, en cualquier tipo que genere valor para las organizaciones.

\section{Análisis de modelos aplicados a las variables}

Son múltiples los modelos y las teorías en el estudio de las variables de capital intelectual y de innovación en turismo. Los modelos más citados para el estudio del capital intelectual son el de balance scorecard de Kaplan y Norton (1996), el Canadian Imperial Bank de Davenport y Prusak (1998), el de ciudadanía digital de López (2010), el integral de gestión del conocimiento de Wiig (1993), el participativo de gestión del conocimiento de Holsapple y Joshi (2002), el inteligente del conocimiento (Megico) diseñado por Del Moral et al. (2007), el de gestión del 
conocimiento de Nonaka y Takeuchi (1995), el Skandia, el de KMAT y el modelo de la Universidad de West Ontario de Bontis (1998).

Otros modelos más enfocados a la innovación son el de gestión del conocimiento como fuente de innovación, propuesto por Nagles (2007), el Intertwining Model o modelo entrelazado, y el de la triple hélice, esbozado por Etkowitz y Leydesforff (1996).

\section{Análisis de las teorías de capital intelectual e innovación}

Más que un modelo de análisis, en esta sección se expondrán las principales perspectivas teóricas que acompañan a los trabajos en el campo de investigación del capital intelectual y la innovación enfocados en el turismo de salud.

La teoría de los recursos y capacidades (Grant, 1991, citado por González, 2002), Estrada y Dutrénit (2007), Ochoa, Prieto y Santidrián (2012); Mercado, Demuner y Fierro (2012). Todos estos autores comentan que los recursos y las capacidades que posea y controle una empresa conformarán y delimitarán las alternativas estratégicas. Esta teoría explica la relevancia de los recursos intangibles, escasos, difíciles de imitar e insustituibles.

En la teoría del capital intelectual (Edvinsson y Malone, 1998), utilizada como base por Ochoa, Prieto y Santidrián (2012) y por Bontis, Chua y Richardson (2000), se consideran tres tipos de recursos intangibles ligados a la ventaja competitiva de la firma. Es decir, trata exclusivamente del conocimiento que es creado y acumulado en los tres componentes de capital de la empresa: la gente (capital humano), las relaciones sociales (capital social) y los sistemas y procesos (capital organizacional o relacional).

Por su parte, la teoría de las capacidades dinámicas (Teece, 1998), también expuesta por Ochoa, Prieto y Santidrián (2012), muestra la habilidad de la empresa para integrar, construir y reconfigurar competencias internas y externas en dirección a los cambios ambientales rápidos. El término dinámico alude a la capacidad de renovación de competencias, así como al logro de congruencia con ambientes cambiantes de negocio.

La teoría de los stakeholders (Clarkson, 1995 citado por Ochoa, Prieto y Santidrián, 2012); Chen (2014) y Hoarau y Kline (2014). En este caso, los stakeholders son personas o grupos que tienen (o pueden demandar) derechos de 
propiedad, así como intereses en una empresa y sus actividades y clasifican a los stakeholders como primarios (accionistas, inversores, empleados, clientes, proveedores, gobierno y comunidad) y secundarios (aquellos que se ven influenciados por la corporación pero no se comprometen en transacciones con la misma, tales como ambientalistas, representantes de los medios o defensores de los clientes).

La teoría de la agencia, citada por Ochoa, Prieto y Santidrián (2012), se cimienta en dos enfoques: el normativo y el positivo. El primero incluye los intangibles dentro de los contratos, mientras que el segundo establece que los sistemas de información del capital intelectual son instrumentos valiosos para alentar la utilización eficiente del conocimiento en el seno de la empresa y proporcionar información relevante para la toma de decisiones.

Más que una teoría, el enfoque en clústeres es el más demandado en los trabajos sobre innovación. Autores como Ivars, Vera y Acebal (2014); Yeoh, Othman y Ahmad (2013); Viladrich y Baron-Faust (2014); Arsezen-Otamis y Yuzbasioglu (2013); Jin, Weber y Bauer (2012); Carlisle et al. (2013); Joukes, Lourenço-Gomes y Marta-Costa (2013); Hoarau y Kline (2014); Novelli, Schmitz y Spencer (2006); Thomas y Wood (2014), y Richards (2011) la utilizan como base y enfoque de sus trabajos de investigación. Desde hace tiempo se atribuye un funcionamiento positivo de determinadas externalidades que favorecen la innovación. Generalmente este fenómeno es conocido como clúster, y se refiere a concentraciones de empresas en ciertas localidades geográficas. El éxito de un clúster radica en gran medida en el equilibrio entre la competencia y la cooperación de los agentes que participan en él.

\section{Tipos de investigación e instrumentos de recolección de la información}

Las investigaciones consultadas sobre capital intelectual e innovación en turismo de salud son en su mayoría ensayos, artículos de opinión y estudios de casos; solo seis de cr (24\%) y cinco de innovación (33\%) equivalen a investigaciones aplicadas. Del total de investigaciones aplicadas en CI, cuatro (66.6\%) son de tipo cualitativo (Alizadeh, 2012; Marulanda y López, 2013; Estrada y Dutrénit, 2007; Bontis, Chua y Richardson, 2000) y dos (33.3 \%) de tipo 
cuantitativo (Heredia, 2009; Huang et al., 2008). Las primeras buscan comprender, por medio de significados y desde una perspectiva más holística, el conjunto de cualidades interrelacionadas que caracterizan a un fenómeno particular.

Marulanda y López (2013) y Bontis, Chua y Richardson (2000) utilizaron un cuestionario a partir de escalas de Likert, con dimensiones como el análisis organizacional, las competencias, las prácticas, los procesos y las tecnologías de la información, lo que les permitió comparar el estado de las empresas del mismo sector. De la misma forma, Estrada y Dutrénit (2007) emplean el cuestionario, realizando preguntas cerradas de tipo cualitativo categórico en entrevistas cara a cara. Los temas que incluyeron van desde el perfil de la empresa y el propietario, sus estrategias, capacidades tecnológicas, actividades de aprendizaje, aseguramiento de la calidad e innovación y mecanismos de financiamiento, hasta la valoración del entorno, las vinculaciones con otros agentes y las políticas públicas. Por su parte, Alizadeh, (2012) utiliza un modelo que le permite analizar variables como los factores de mercado, el aspecto humano, la propiedad intelectual y la estructura. Algunos autores hacen inferencias matemáticas de las dimensiones del capital intelectual y usan métodos cuantitativos, pero se trata solo de dos de los trabajos revisados (Heredia, 2009; Huang et al., 2008).

Un trabajo interesante y que difiere de los métodos tradicionales de investigación sobre el capital intelectual es el de Huang et al. (2008), que utiliza un estudio empírico de relación causal para observar las correlaciones entre los elementos que forman el modelo de balance scorecard: las perspectivas financieras, del aprendizaje y el crecimiento de la firma, de los clientes y la de procesos internos.

Por otro lado, la mayoría de las investigaciones sobre innovación, específicamente en turismo, son de tipo cualitativo (Hoarau y Kline, 2014; Carlisle et al., 2013; Yeoh, Othman y Ahmad, 2013; Thomas y Wood, 2014, Huang et al., 2008). Las técnicas utilizadas por Hoarau y Kline (2014) pueden definirse como una matriz de interpretación de enfoques que intentan describir, decodificar, traducir y llegar a un acuerdo a través del análisis, es decir, con la frecuencia de los fenómenos que ocurren. Su investigación se sitúa entre los estudios deductivos e inductivos, pero no trata de probar una teoría ni de desarrollar alguna nueva, sino, más bien, contribuir a la construcción de alguna mediante la interacción dialéctica entre los estudios de campo y la teoría existente. Los trabajos de 
estos investigadores incluyeron la recolección de datos por medio de entrevistas, revisiones de reportes públicos y observación participante. Esta triangulación de instrumentos y métodos le dio credibilidad y validación al estudio. Las entrevistas versaron en esencia sobre sustentabilidad, innovación, cooperación y uso del conocimiento.

Carlisle et al. (2013) aplican el modelo de la triple hélice de análisis estratégico, por lo que establecen tres etapas de investigación. En la primera fase utilizan el instrumento de la observación participante, en la segunda, los investigadores participan de manera activa con los sujetos de estudio, y en la tercera conviven con ellos para resolver problemas y aplicar activamente el modelo de análisis estratégico.

Al igual que los trabajos anteriores, las investigaciones de Thomas y Wood (2014) están encaminadas a un método estratégico de innovación en el turismo. Estos autores realizaron entrevistas informales con el objetivo de definir las preguntas para un cuestionario más formal. Este instrumento con una escala de Likert se aplicó a 100 gerentes de hoteles. Las dimensiones fueron: servicios de acceso, transformación, asimilación y explotación.

Huang et al. (2008) aplican el modelo de balance scorecard a los trabajos sobre innovación y capital intelectual, por lo que, en lugar de elegir un instrumento de recolección de datos, introducen la información en cuatro grupos de análisis de la industria: los factores financieros, la dimensión de los clientes, las relaciones internas del negocio y el crecimiento y aprendizaje de la firma.

Yeoh, Othman y Ahmad (2013) recurren al cuestionario como instrumento por excelencia para obtener la información, y lo envían a clínicas y hospitales del área de estudio. Estos autores tratan de determinar dimensiones como: información demográfica, tipo de padecimiento, días de estancia, cómo obtuvo información del servicio médico, el costo estimado, entre otros datos relevantes.

Vale hacer notar que son pocos los artículos de investigación aplicada para hacer una inferencia general del comportamiento de los estudios de las variables de cr e innovación. Sin embargo, fueron suficientes para obtener datos que permiten relacionar las variables estudiadas e iniciar un proyecto más amplio que incluya estas variables y un exhaustivo y mejor dirigido "análisis de la literatura”. 


\section{Discusión}

La información vertida en la etapa anterior se puede analizar numéricamente, que del total de las definiciones encontradas sobre capital intelectual, $53 \%$ tiene una tendencia hacia la gestión del conocimiento, 33 \% un origen financiero y contable, y $14 \%$ se centra en relacionar el ci como una fuente de ventajas competitivas, que corresponde a los estudios más recientes (de 2009 a 2014).

Asimismo, donde se incluye el término innovación se puede concluir que se trata de un concepto antiguo y que toma forma a partir de la Revolución Industrial, por lo que sus primeros enfoques (13\%) la describen como cambios en los procesos mecánicos y en la maquinaria de producción, 35\% de los artículos incorporan la innovación en las ideas, 9 \% la enfoca como la modificación en los procesos de producción y en los sistemas, y $43 \%$ la sitúa como elemento vital en los procesos para la competitividad.

Respecto al análisis de los objetivos, se recuerda que 25 trabajos corresponden a la variable de ci y 15 trabajos a la variable innovación. De esta forma, los objetivos de los estudios versaron en $16 \%$ sobre el capital intelectual como parte indispensable de la gestión del conocimiento, $20 \%$ de los trabajos incluyen los componentes de medición del cr, $12 \%$ mantienen como temática los modelos de medición, y en $20 \%$ el objetivo es mostrar la relación del ci como capacidad interna que mejorará el comportamiento organizacional de las empresas. En $12 \%$ de las revisiones de literatura más actuales se observa, por su parte, una inclinación a centrar el cr en la producción de ventajas competitivas, y en $20 \%$ como fuente de creación de innovación para las organizaciones.

De los 15 estudios sobre la variable innovación, 13 \% la identifica como elemento generador de ventajas competitivas sostenibles, $34 \%$ enlaza esta variable con el cI, y 53 \% señala en sus objetivos el estudio de la innovación en el turismo.

Asimismo, puede concluirse que de los artículos donde se mencionan los componentes del capital intelectual, $7 \%$ considera que estos son los activos del mercado, activos de propiedad intelectual, activos centrados en el individuo y activos de infraestructura, $7 \%$ sostiene un enfoque contable y los subdivide en activos físicos y activos intangibles; por otro lado, $7 \%$ destaca las tecnologías de la información, y los divide en recursos humanos, recursos de información y una red de recursos informáticos y telemáticos, $14 \%$ considera que los componentes 
del ci son recursos humanos, de relaciones, infraestructura física, prácticas, rutinas y propiedad intelectual. Para finalizar, $65 \%$ coincide en establecer como elementos indispensables el capital humano, el estructural y el relacional.

Sin embargo, los componentes de la innovación solo se mencionaron en cuatro trabajos, de tal forma que son insuficientes para hacer inferencias concluyentes; queda manifestar que, en general, las innovaciones pueden darse en los productos, en los procesos, en la gestión, en las acciones de mercadotecnia, en los suministros, en nuevos mercados y en las instituciones.

Ahora bien, en cuanto a las teorías que sustentan a la variable cI, de los diez trabajos que citan en su contenido la visión epistemológica, 40 \% se basa en la teoría de los recursos y las capacidades, 20 \% cita la teoría del capital intelectual, $20 \%$ se apoya en la de los stakeholders, $10 \%$ en la teoría de la agencia y otro $10 \%$ tomó como fundamento la teoría de las capacidades dinámicas.

Por último, para la variable innovación se revisaron 12 artículos, todos los cuales se basan en la teoría de clústeres.

\section{Conclusiones}

La revisión de la literatura es uno de los ejercicios más importantes porque permite los siguientes objetivos: identificar los temas de investigación que no han sido estudiados lo suficiente o los enfoques que necesitan abarcarse, las variantes en los estilos y perfiles que retoma cada autor y la información más novedosa sobre las variables; determinar y redactar los enfoques teóricos y los aspectos del marco conceptual; revisar los enfoques metodológicos; redescubrir nuevas líneas de investigación sugeridas por los autores, entre otras posibilidades relevantes.

Asimismo, la elaboración de las tablas matrices que incluyan el desglose de la información principal hace pertinente su valoración, el control y el análisis de las fuentes elegidas para un proyecto de investigación. Es sin duda un trabajo que se ha realizado paso a paso, descubriendo en el camino su trascendencia y conformando un ciclo aún no terminado.

En resumen, tras llevar a cabo las revisiones sistémicas de los 25 artículos básicos elegidos de la variable capital intelectual y los 15 de la variable innovación, puede destacarse que la gestión del capital intelectual, sea humano, 
relacional o estructural, genera las posibilidades de innovación en distintos frentes -ya sea en los productos, procesos, métodos de organización o estrategias de mercadotecnia en diferentes tipos de organizaciones- y pone de relieve el uso de la innovación para acrecentar la eficiencia en las empresas de servicio turístico de salud.

La mayoría de las investigaciones aplicadas recomienda utilizar estudios cualitativos que permitan revisar, en una forma más holística, qué, cómo o por qué ocurren ciertos fenómenos. Aunque tienen la limitante de que sus cuantificaciones son apreciaciones de cada investigación -y difícilmente pueden hacerse aseveraciones concluyentes como en las investigaciones cuantitativas- sí es factible establecer un panorama y reflejar una realidad del fenómeno. Estos estudios sugieren el uso de instrumentos de recolección de datos como los cuestionarios, las entrevistas semiestructuradas, las observaciones libres y participantes.

Por último, es necesario reconocer que la presente revisión sistémica se circunscribe a los artículos elegidos por el interés en el manejo de las variables y por su enfoque en la innovación en turismo, lo que ofrece una mirada incompleta sobre el fenómeno de estudio. Sin embargo, permite observar con claridad los principales enfoques en los que se ha trabajado el fenómeno del cI y la innovación y la dirección que han seguido ambos temas hacia el logro de ventajas competitivas sostenibles, cualquiera que sea el tipo de organización o actividad económica de la que se trate.

No queda más que sugerir una revisión más exhaustiva del fenómeno con información actualizada, útil, confiable y de alto impacto, que genere confianza y aceptación en los círculos de investigación. Además, conviene indagar en estudios en diferentes idiomas, lugares y contextos que posibiliten hacer comparaciones y brinden la confiabilidad y validez requerida para hacer inferencias reales sobre el fenómeno de estudio.

\section{Fuentes consultadas}

Alizadeh, E. (2012). "Identification, Measurement and Management of Intellectual Capital is a Vital Importance Issue for the Survival of Organization in the Field of Competition”. Life Science Journal, 9 (4), 761-765. 
Amaya, K., Iriarte, M. y Perozo, D. (2006). “Gestión del conocimiento como capacidad interna”. Telos, 8 (2), 250-265.

Arsezen-Otamis, P. y Yuzbasioglu, N. (2013). "Analysis of Antalya Tourism Cluster Perceived Performance with Structural Equation Model”. Procedia. Social and Behavioral Sciences, 99 (6), 682-690.

Blichfeldt, B. (2009). "Innovation and Entrepreneurship in Tourism: The Case of a Danish Caravan Site”. Pasos. Revista de Turismo y Patrimonio Cultural, 7 (3), 415-431.

Bontis, N. (1998 a). "Intellectual Capital: An Exploratory Study that Develops Measures and Models”. Management Decision, 36 (2), 63-76.Bontis, N., W. Chua y S. Richardson (2000). "Intellectual Capital and Business Performance in Malaysian Industries”. Journal of Intellectual Capital, 1 (1), 85-100.

(1998 b). Intellectual capital: an exploratory study that develops measures and models. Management Decision, 36(2), pp. 63-76.

Brooking, A. (1997). Intellectual Capital. Londres: International Thomson Business Press.Carbone, F. et al. (2012). "Open Innovation in an Enterprise 3.0 Framework: Three Case Studies”. Expert Systems with Applications, 39 (10), 8929-8939.

Carlisle, S. et al. (2013). "Supporting Innovation for Tourism Development through Multi-Stakeholder Approaches: Experiences from Africa”. Tourism Management, 35, 59-69.

Chen, J. (2014). Tourism stakeholders attitudes towards sustainable development: A case in the Artic. Journal of Retaling and Consumer Services, pp. 1-6.

Chen, M., Cheng, J. \& Hwang, Y. (2005). "An Empirical Investigation of the Relationship between Intellectual Capital and Firm's Market Value and Financial Performance”. Journal of Intellectual Capital, 6 (2), 159-176. Chen, J. y Tsou T. (2007). "Information Technology Adoption for Service Innovation Practices and Competitive Advantage: The Case of Financial Firms”. Information Reseach [en línea], 12 (3). Disponible en: http:// www.informationr.net/ir/12-3/paper314.html

Chong, A, y Zhou, L. (2014). "Demand Chain Management: Relationships between External Antecedents, Web-Based Integration and Service Inno- 
vation Performance”. International Journal of Production Economics, 154, 48-58.

Correa, R., Rodrigues, R. y Pereira, H. (2007). "Gestão do conhecimento ou gestão de organizações da era do conhecimento? Um ensaio teóricoprático a partir de intervenções na realidade brasileira”. Perspectivas em Ciência da Informação, 12 (1), 5-24.

Damanpour, F. (1990). "Innovation Effectiveness, Adoption and Organizational Performance”, en M. West y J. Farr (eds.). Innovation and Creativity at Work. Nueva York: John Wiley \& Sons, 125-141.

------- y Gopalakrishnan, S. (1997). “A Review of Innovation Research in Economics, Sociology and Technology Management”. Omega. The International Journal Management Science, 25 (1), 15-28.

Darceles, M. (2007). “Gestión del conocimiento y creatividad”. Revista Escuela de Administración y Negocios, 61, 97-102.

Del Moral, A., Pazos, J., Rodríguez, E., Rodríguez, P., \& Suarez, S. (2007). Gestion del Conocimiento. Madrid: Thomson.

Di Domeneico, A., De Bona, G. y Fernández, O. (2003). La inteligencia en acción: gestionar por el conocimiento. Biblios, 4(15), pp. 12-20.

Drucker, P. (1985). La innovación y el empresario innovador. Barcelona: Edhasa.

Dzinkowski, R. (1998). The Measurement and Management of Intellectual Capital: An Introduction. Nueva York: Financial and Management Accounting Committee-International Federation of Accountants. Consultado el 12 de septiembre de 2014, disponible en: http://www. ciberconta.unizar.es/ftp/pub/docs/IntellCapital_IFAC.pdf

Edvinsson, L. y Malone, M. (1998). El capital intelectual. Bogotá: Norma.

Estrada, S. y Dutrénit, G. (2007). "Gestión del conocimiento en pymes y desempeño competitivo”. Engevista, 9 (2), 129-148.

Etzkowitz H. y Leydesdorff L., 1996, Emergence of a triple helix of Universityindustry-government relations. Science and Public Policy, (23), pp. 279-286.

Eurostat/ocDe (2006). Manual de Oslo. Guía para la recogida e interpretación de datos sobre innovación. Madrid: Oficina de Estadística de las Comunidades Europeas/Organización para la Cooperación y el Desarrollo 
Económicos/Grupo Tragsa.

Fagerberg, J., Mowery, D. y Nelson, R. (2006). The Oxford Handbook of Innovation. Oxford: Oxford University Press.

Fundación Cotec (2002). Informe Cotec. Tecnología e innovación en España. Madrid: Fundación Cotec para la Innovación Tecnológica.

Funes, Y. y Hernández, C. (2001). "Medición del valor del capital intelectual”. Contaduría y Administración, 203, 45-58.

García-Ayuso, M. y Larrinaga, C. (2004). "El lado oculto de los intangibles: activos y pasivos ligados a la sostenibilidad”. Harvard Deusto Finanzas y Contabilidad, 57, 34-41.

García, T. (2005). "Diseño de un modelo para la medición del capital intelectual de empresas de manufactura en el Perú”. Revista de la Facultad de Ingeniería Industrial, 8 (2), 33-41.

González, L. (2002). "Gestión del conocimiento y gestión de recursos humanos: una convergencia necesaria”. Revista de Psicología del Trabajo y de las Organizaciones, 18 (2-3), 177-213.

González-Loureiro, M. y Figueroa, P. (2013). “El papel del capital relacional en el éxito de los clúster: el caso del Clúster de Turismo Termal de Galicia (AEITEGAL)", en V. Joukes, L. Lourenço-Gomes y A. Marta-Costa (eds.). Sustainable Health and Wellness Destinations. Proceedings of the International Conference Held in Chaves (Portugal), 13-15/10/2011. Vila Real: Universidade de Trás-os-Montes e Alto Douro, 151-178.

Hall, R. (1992). The strategic analysis of intangible resources. Strategic Management Journal. 13(2), pp. 135-144.

Heredia, J. (2009). Influencia del capital intelctual en la competitividad de los hoteles. Conciencia Tecnológica, (37), pp. 20-25.

Hoarau, H. y Kline, C. (2014). "Science and Industry: Sharing Knowledge for Innovation". Annals of Tourism Research, 46, 44-61.

Holsapple, C., y Joshi, K. (2002). Knowledge management: A three-fold framework. Information Society, 18.

Huang, H. et al. (2008). "Strategic linkage process and value-driven system: A dynamic analysis of high-tech firms in a newly-industrialized country". Expert Systems with Applications, 36 (2), 3965-3974.

Inche, J. y Álvarez. J. (2007). “Indicadores de gestión del conocimiento”. Cien- 
cia en su PC [en línea], 2. Disponible en: http://www.redalyc.org/ pdf/1813/181320217003.pdf

Ivars, J., Vera, J. y Acebal, A. (2014). "Políticas de innovación en turismo y desarrollo de clusters: la percepción gerencial en el programa Agrupaciones Empresariales Innovadoras (AEIS)". Cuadernos de Turismo, 33, 97-120.

Jin, X., Weber, K. y Bauer, T. (2012). "Impact of Clusters on Exhibition Destination Attractiveness: Evidence from Mainland, China”. Tourism Management, 33 (6), 1429-1439.

Johannessen, J., Olsen, B. y Lumpkin, G. (2001). "Innovation as Newness: What is New, How New, and New to Whom?”. European Journal of Innovation Management, 4 (1), 20-31.

Joukes, V., Lourenço-Gomes, L. y Marta-Costa, A. (2013). Sustainable Health and Wellness Destinations. Proceedings of the International Conference Held in Chaves (Portugal), 13-15/10/2011. Vila Real: Universidade de Trás-os-Montes e Alto Douro.

Kaplan, R., \& Norton, D. (1996). The Balanced Scorecard: Translating Strategy Into Action EEUU: Harvard Business School Press.

Klein, D. (1998). The Strategic Management of Intellectual Capital. Nueva York: Butterworth-Heinemann.

y Prusak, L. (1994). Characterizing Intellectual Capital. Boston: Ernst \& Young LLP/Center for Business Innovation (Working Paper).

López, M. (2010). Ciudadanía Digital, un modelo de implantación en la región de Manizales y Caldas, Colombia. Madrid: Universidad pontificia de Salamanca.

Madrigal, B. (2009). “Capital humano e intelectual: su evaluación”. Observatorio Laboral. Revista Venezolana, 2 (3), 65-81.

Magaña, I. (2014). El capital humano especializado en la empresa turística a través de competencias cualitativas hacia la innovación. Revista de investigación en turismo y desarrollo local. 7(16), pp. 1-25.

Marr, B, (2004), "Measuring and Benchmarking Intellectua “, International Journal, Vol. 11, No. 6, pp.559-570.

Martínez, C. (2004). “Gestión y creación de conocimiento”. Innovar. Revista de Ciencias Administrativas y Sociales, 23, 13-23. 
McElroy, M. (2002). The New Knowledge Management - Complexity, Learning and Sustainable Innovation. Londres: Butterworth-Heinemann.

Medina, C. y Espinosa, M (1994). "La innovación en las organizaciones modernas”. Gestión y Estrategia [en línea], 5, 54-63. Disponible en: http:// gestionyestrategia.azc.uam.mx/index.php/articulos?format = raw\&tas $\mathrm{k}=$ download\&fid $=512[2014,7$ de septiembre $]$.

Mercado, P., Demuner, M. Y Fierro, E. (2012). Innovación no tecnológica en empresas auxiliares del sector salud. Caso de estudio de un laboratorio clínico. Revista Interamericana de Psicología Ocupacional, 31(2), pp. 101-119.

Muñoz, M., Aguado, D. y Lucía, B. (2003). "El largo camino hacia la gestión del conocimiento". Revista de Psicología del Trabajo y de las Organizaciones, 19 (2), 199-214.

Marulanda, C. y López, M. (2013). “La gestión del conocimiento en las Pymes de Colombia”. Revista Virtual Universidad Católica del Norte, 38, 158170.

Nagles, N. (2006). Productividad: una propuesta desde la gestión del conocimiento. Revista Escuela de Administración de Negocios, (58), pp. 87105.

(2007). "La gestión del conocimiento como fuente de innovación". Revista Escuela de Administración de Negocios, 61, 77-87.

Navarro, E. (2005). "La innovación como concepto estratégico". Revista Improven Empresarial [en línea]. Disponible en: http://www.improvenconsultores.com [2014, 9 de septiembre].

Nonaka, I. y Takeuchi, H. (1995). The Knowledge-Creating Company: How Japanese Companies Create the Dynamics of Innovation. Oxford: Oxford University Press.

Novelli, M., Schmitz, B. y Spencer, T. (2006). "Networks, Clusters and Innovation in Tourism: A uk Experience”. Tourism Management, 27 (6), 1141-1152.

Ochoa, M., Prieto, M. y Santidrián, A. (2012). “Una revisión de las principales teorías aplicables al capital intelectual”. Revista Nacional de Administración, 3 (2), 35-48.

Orozco, L., Chavarro-Bohórquez, D. y Rivera, H. (2007). “Estrategia y conoci- 
miento en la gestión organizacional”. Revista Universidad y Empresa, 6 (13), 37-58.

Perozo, M. (2004). "Gestión del conocimiento en la capacitación para la innovación”. Revista Venezolana de Análisis de Coyuntura, X (2), 117-129.

Richards, G. (2011). "Creativity and Tourism. The State of the Art”. Annals of Tourism Research, 38 (4), 1225-1253.

Rivas, L. y Flores, B. (2007). "La gestión del conocimiento en la industria automovilística”. Estudios Gerenciales, 23 (102), 83-100.

Roos, G. et al. (1997). Intellectual Capital - Navigating in the New Business Landscape. Nueva York: New York University Press.

Sánchez, A., Melián, A. y Hormiga, E. (2007). El concepto de capital intelectual y sus dimensiones. Investigaciones Europeas de Dirección y Economía de la Empresa, 13(2), pp. 97-111.

Sánchez-Meca, J. y Botella. J. (2010). "Revisiones sistemáticas y meta-análisis. Herramientas para la práctica profesional”. Papeles del Psicólogo, 31 (1), 7-17.

Schumpeter, J. (1939). Business Cycles: A Theoretical, Historical, and Statistical Analysis of the Capitalist Process. Nueva York: McGraw-Hill.

Smith, A. (1994). Investigación sobre la naturaleza y causas de la riqueza de las naciones. México: Fondo de Cultura Económica.

Stewart, A. (1997). Intellectual Capital. The New Wealth of Organizations. Nueva York: Bantam Double Day Publishing.

Sveiby, K. (1997). The New Organizational Wealth: Managing and Measuring Knowledge-Based Assets. San Francisco: Berrett Koehler.

Teece, D. (1998). "Capturing Value from Knowledge Assets: The New Economy, Markets for Know-How, and Intangible Assets". California Management Review, 40 (3), 55-79.

Trillo, M. y Pedraza, J. (2011). "La influencia de la innovación en el capital intelectual de la empresa. Propuesta de un modelo”, en J. Ayala Calvo y Grupo de Investigación FEDRA (coords.). Conocimiento, innovación y emprendedores: camino al futuro. La Rioja: Universidad de La Rioja, 1419-1431.

Thomas, R. y Wood, E. (2014). "Innovation in Tourism: Re-Conceptualising and Measuring the Absorptive Capacity of the Hotel Sector". Tourism Management, 45, 39-48. 
Tushman, M. y Nadler, D. (1986). “Organizing for Innovation”. California Management Review, 28 (3), 74-92.

Ulrich, D. (1998). "Intellectual Capital Equals Competence x Commitment”. Sloan Management Review, 39 (2), 15-27.

Urrútia, G. y Bonfill, X. (2010). “Declaración PRISMA: una propuesta para mejorar la publicación de revisiones sistemáticas y metaanálisis”. Medicina Clínica, 135 (11), 507-511.

Van de Ven, A. (1986). "Central Problems in the Management of Innovation”. Management Science, 32 (5), 590-607.

Valhondo, D. (2004). Gestión del conocimiento. Del mito a la realidad [en línea]. Madrid: Ediciones Díaz de Santos. Disponible en: http:site.ebrary.com/ lib/bibliotecaeansp/Doc?id $=10069401 \& p p g=113$ [2014, 8 de septiembre].

Vandenbroucke, J., Von, E., Altman, D., Gotzsche, P., Mulrow, C., Pocock, S., Poole, C., Schlesselm, J. \& Egger, M. (2009). Mejorar la comunicación de estudios observacionales en epidemiología (STROBE): explicación y elaboración.

Viedma, J. (2007). "In Search of an Intellectual Capital Comprehensive Theory”. Electronic Journal of Knowledge Management, 5 (2), 245-256.

Viladrich, A. y Baron-Faust, R. (2014). "Medical Tourism in Tango Paradise: The Internet Branding of Cosmetic Surgery in Argentina”. Annals of Tourism Research, 45, 116-131.

Wiig, K. (1993). Knowledge Management Foundations: Thinking about Thinking-how Pleople and Organizations Create, Represent and Use of Knowledge. Arlington: Schema Press.

Yeoh, E., Othman, K. y Ahmad, H. (2013). "Understanding Medical Tourist: Word-of-Mouth and Viral Marketing as Potent Marketing Tools”. Tourism Management, 34, 196-201.

Youndt, M., Subramaniam, M. y Snell, S. (2004). "Intellectual Capital Profiles: An Examination of Investments and Returns”. Journal of Management Studies, 41 (2), 335-361. 